\title{
The Effect of DA-6034 on Intestinal Permeability in an Indomethacin- Induced Small Intestinal Injury Model
}

\author{
Dong Shin Kwak, Oh Young Lee, Kang Nyeong Lee, Dae Won Jun, Hang Lak Lee, Byung Chul Yoon, and Ho Soon Choi
}

Department of Internal Medicine, Hanyang University School of Medicine, Seoul, Korea

Background/Aims: DA-6034 has anti-inflammatory activities and exhibits cytoprotective effects in acute gastric injury models. However, explanations for the protective effects of DA-6034 on intestinal permeability are limited. This study sought to investigate the effect of DA-6034 on intestinal permeability in an indomethacin-induced small intestinal injury model and its protective effect against small intestinal injury. Methods: Rats in the treatment group received DA-6034 from days 0 to 2 and indomethacin from days 1 to 2 . Rats in the control group received indomethacin from days 1 to 2. On the fourth day, the small intestines were examined to compare the severity of inflammation. Intestinal permeability was evaluated by using fluorescein isothiocyanate-labeled dextran. Western blotting was performed to confirm the association between DA-6034 and the extracellular signal-regulated kinase (ERK) pathway. Results: The inflammation scores in the treatment group were lower than those in the control group, but the difference was statistically insignificant. Hemorrhagic lesions in the treatment group were broader than those in the control group, but the difference was statistically insignificant. Intestinal permeability was lower in the treatment group than in the control group. DA-6034 enhanced extracellular signal-regulated kinase expression, and intestinal permeability was negatively correlated with ERK expression. Conclusions: DA-6034 may decrease intestinal permeability in an indomethacin-induced intestinal injury model via the ERK pathway. (Gut Liver 2016;10:406-411)

Key Words: DA-6034; Intestinal permeability; Small intestinal injury model; Leaky gut syndrome

\section{INTRODUCTION}

The epithelial layer of the gastrointestinal tract serves as one of the primary interfaces with the outside world. The mucosal surface of the intestinal epithelium is in constant contact with innumerable microbes and their metabolites. The leaky gut syndrome has received increasing attention in the recent past. The leaky syndrome is simply a phenomenon of passage of lipopolysaccharide or toxins of gram-negative bacteria into the blood stream due to disintegration of intestinal mucosa, which causes bacteremia, endotoxemia, and systemic inflammation. Increased intestinal permeability is known to be associated with several circumstances; the leaky gut syndrome is associated with the long-term use of nonsteroidal anti-inflammatory drug (NSAID), which is an essential pathogenesis of inflammatory bowel disease (IBD) and irritable bowel syndrome (IBS). ${ }^{1-4}$ It also contributes to the expression and progression of the alcoholic liver disease and chronic liver disease as well as congestive heart failure. ${ }^{5,6}$ Moreover, the leaky gut syndrome has been viewed as an important pathogenesis of diabetic mellitus and obesity in relation to insulin resistance. ${ }^{7,8}$

To treat and prevent the increase of intestinal permeability, some studies have been performed. While rebamipide, probiotics, and proton pump inhibitor have been studied, they elucidated protective effects on intestinal permeability. ${ }^{9-11}$ DA6034, which is an active derivative of Artemisia extracts in plants, exhibited not only a gastroprotective effect in several gastric injury models, but also indicated anti-inflammatory effect by inhibiting nuclear factor kappa-light-chain-enhancer of activated B cells $(\mathrm{NF}-\kappa \mathrm{B}){ }^{12,13}$ However, there are only limited explanations about the protective effects of DA-6034 on intestinal permeability. The study aims at evaluating the effect of DA-6034 on intestinal permeability, using fluorescein isothiocyanate-labeled dextran. It also compares the inflammation severity of the treatment group with that of the control group using hematoxylin and eosin (H\&E) stain. The Western blot analysis was also carried out to confirm the association of DA-6034 with extracel-

Correspondence to: Oh Young Lee

Department of Internal Medicine, Hanyang University School of Medicine, 222 Wangsimni-ro, Seongdong-gu, Seoul 04763, Korea

Tel: +82-2-2290-8343, Fax: +82-2-2298-8314, E-mail: leeoy@ hanyang.ac.kr

Received on June 4, 2015. Revised on July 10, 2015. Accepted on July 10, 2015.

pISSN 1976-2283 eISSN 2005-1212 http://dx.doi.org/10.5009/gnl15251

@ This is an Open Access article distributed under the terms of the Creative Commons Attribution Non-Commercial License (http://creativecommons.org/licenses/by-nc/4.0) which permits unrestricted non-commercial use, distribution, and reproduction in any medium, provided the original work is properly cited. 
lular signal-regulated kinase (ERK) pathway in the prevention of the leaky gut syndrome.

\section{MATERIALS AND METHODS}

\section{Animal and reagent}

Orient Bio, Korea provided 36 Sprague-Dawley rats, weighing $250 \mathrm{~g}$ at 6 weeks. The rats were housed in animal facilities with $50 \% \pm 5 \%$ humidity, $23^{\circ} \mathrm{C} \pm 2^{\circ} \mathrm{C}$ temperature and a $12: 12$-hour light-dark cycle and raised with samtako during the experiment. All experiments were performed in accordance with the institutional and national guidelines for the care and use of laboratory animals approved by the Ethics Committee of Hanyang University (HY-IACUC-10-020).

\section{Experimental protocol}

Rats were randomly divided into two groups namely; DA6034/indomethacin and indomethacin group. In the treatment group, rats were orally administered DA-6034 $30 \mathrm{mg} / \mathrm{kg} \mathrm{q} 12$ hours from the day 0 to day 2 with indomethacin $15 \mathrm{mg} / \mathrm{kg} \mathrm{q}$ 12 hours from day 1 to day 2 . In the control group, rats were administered only indomethacin $15 \mathrm{mg} / \mathrm{kg}$ q 12 hours from day 1 to day 2. On day 3, rats were dissected and small intestines were removed immediately (Fig. 1).

\section{Intestinal permeability}

In order to measure intestinal barrier permeability, rats were gavaged with a permeability tracer, FITC-labeled dextran (6 $\mathrm{mg} / 100 \mathrm{~g}$ body weight of FITC-labeled dextran, mol wt. 4,000; Sigma-Aldrich, St. Louis, MO, USA) on day 3. While food and water were withdrawn 4 hours before the gavage. Four hours after gavage, blood was withdrawn by cardiac puncture 4 hours after the gavage. Fluorescence intensity of the serum samples was measured using a Victor 3 spectrophotometer (excitation 490 nm, emission 525 nm, Cytofluor 2300; PerkinElmer, Waltham, MA, USA; Waters Chromatography). The FITCdextran concentrations were determined from standard curves generated by serial dilution of FITC-dextran. Permeability was calculated by the linear regression of sample fluorescence. ${ }^{14}$

\section{Histology}

Histologic examination was carried out on small intestine segments removed from rats of each group. Intestinal segments were immediately injected with 10\% formalin and left in the same fixative solution. After 30 minutes, they were opened along the antimesenteric border, cleaned of fecal content and fixed in a 10\% formalin for 24 hours. Six sections were randomly chosen from each intestinal segment and processed into paraffin. Serial paraffin sections $(4 \mu \mathrm{m})$ were then prepared and stained with $\mathrm{H} \& \mathrm{E}$. An independent observer without previous knowledge of the treatment assessed the histologic damage. The damage was estimated microscopically not only as the sum of the mucosal score but also as the indices of inflammation. The mucosal score was rated on a 4-point scale (0 to 3) according to the criteria established by Chiu et al. ${ }^{15}$ with modifications Moreover, the extent of the hemorrhagic lesion was calculated as well (Table 1).

\section{Western blotting analysis}

To elucidate the mechanism of DA-6034-induced cytoprotective effect, protein expression was examined using the immunoblotting technique. It assumes that the ERK pathway is related

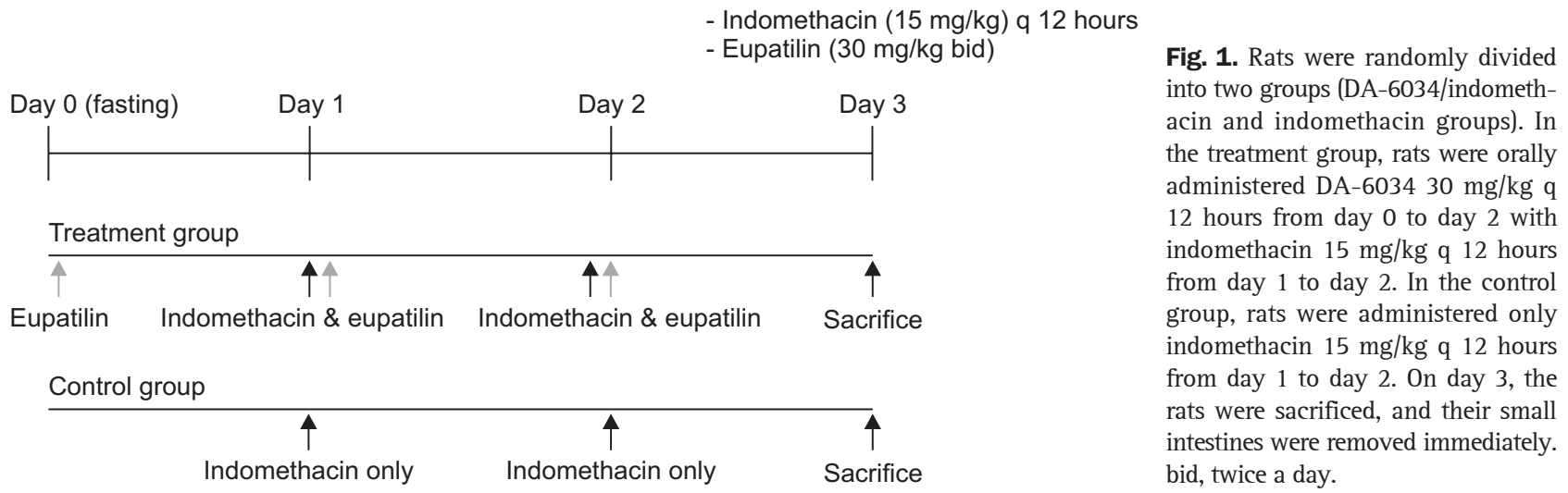

Table 1. Inflammation Score and Histologic Findings

\begin{tabular}{cl}
\hline Inflammation score & Histologic finding \\
\hline 0 & Absence of inflammatory reaction, ulceration or tissue destruction \\
1 & Slight inflammatory reaction but no ulceration or tissue destruction \\
2 & Moderate inflammatory infiltration with mild tissue destruction of villous, crypts but no ulceration \\
3 & Intense inflammatory reaction, presence of ulceration and extensive tissue destruction \\
\hline
\end{tabular}


to the preventive effect of intestinal permeability of DA-6034. The Western blot analysis was employed in confirming the hypothesis. The tissue of small intestine was washed with 4 to 5 $\mathrm{mL}$ of phosphate buffered saline, grinded with homogenizer and incubated for 10 minutes at $0^{\circ} \mathrm{C}$. Then they were centrifuged for 10 minutes and supernatant was gathered. Total protein was acquired by using protein quantification solution (Bio-Rad Laboratories, Hercules, CA, USA). The total proteins were mixed with sodium dodecyl sulfate sample buffer (Tris-Cl [pH6.8] 100 $\mathrm{mM}$, glycerol 20\%, bromophenol blue 0.2\%, SDS 4\%, dithiothreitol $200 \mathrm{mM}$ ) and boiled at $100^{\circ} \mathrm{C}$ for 10 minutes. The samples were then subjected to SDS-polyacrylamide gel and blotted onto a polyvinylidene fluoride membrane (Atto Corp., Tokyo, Japan). The membrane was blocked with Tris-buffered saline and Tween buffer (10 mM Tris, $150 \mathrm{mM} \mathrm{NaCl,} \mathrm{0.1 \%} \mathrm{Tween} \mathrm{20)}$ at room temperature for 60 minutes. Western blotting analysis was carried out using p-ERK antibody, total form ERK antibody (Cell Signaling Technology Inc., Danvers, MA, USA) at $4^{\circ} \mathrm{C}$ overnight. After three washes with TBS-T, the membrane was incubated with anti-rabbit or anti-mouse IgG (IgG-HRP) (Santa Cruz Biotechnology, Santa Cruz, CA, USA) at room temperature for 60 minutes. The signals were visualized using an enhanced chemiluminescence solution (Amersham, Buckinghamshire, UK), according to the manufacturer's instructions.

\section{Statistical analysis}

The t-test was used to test for association among noncategorical variables and correlation test was used to assess association between ERK pathway and intestinal permeability. A p-value $<0.05$ was considered to indicate statistical significance. All statistical analyses were performed using the SPSS version 18 software (SPSS Inc., Chicago, IL, USA)

A

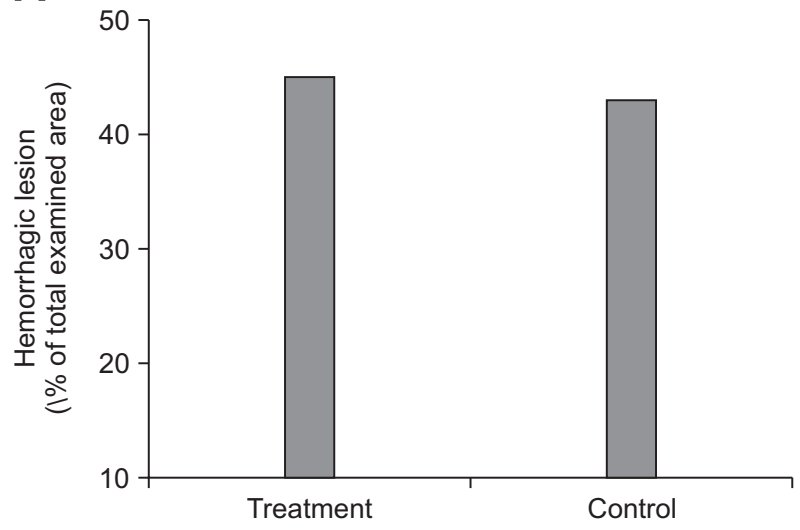

\section{RESULTS}

\section{Effect of DA-6034 on small bowel inflammation}

When the severity of inflammation between two groups was compared macroscopically, the severity of necrosis and color change was more severe in the control group than treatment group. The damage was estimated microscopically as not only the sum of the mucosal score but also the indices of inflammation. The inflammation score was lower in the treatment group, but it was not statistically significant $(2.6 \pm 0.6$ vs $2.9 \pm 0.3$, $\mathrm{p}=0.11$ ) (Fig. 2A). When the extent of hemorrhage was compared, a hemorrhagic lesion in the treatment group was broader than that of the control group but was not statistically significant (45.0 \pm 45.5 vs $43.0 \pm 33.8, \mathrm{p}=0.88$ ) (Fig. $2 B$ ).

\section{Intestinal permeability}

Intestinal permeability evaluated by FITC-labeled dextran was lower in the treatment group compared to control group

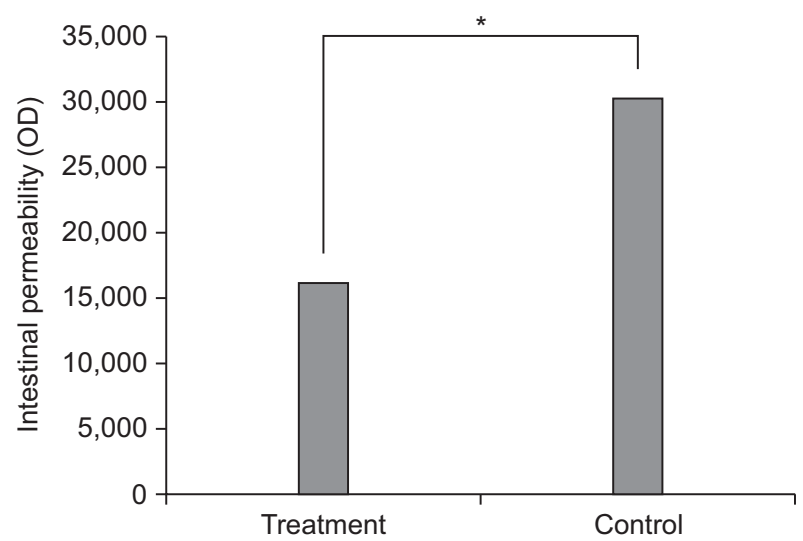

Fig. 3. Intestinal permeability evaluated by using FITC-labeled dextran was lower in the treatment group compared with the control group $(16,204.2 \pm 13,832.1$ vs $30,403.7 \pm 24,012.6, p<0.05)$. ${ }^{*} \mathrm{p}<0.05$.

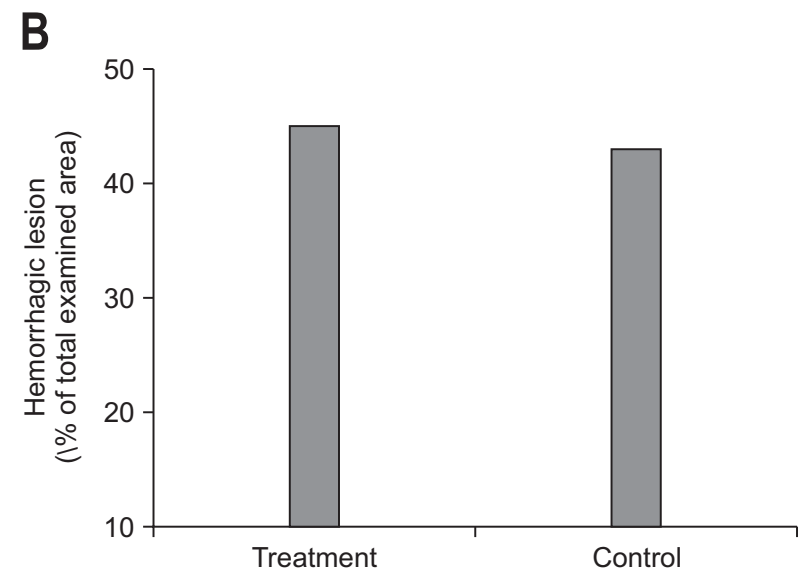

Fig. 2. (A) The inflammation score in the treatment group was lower than in the control group, but the difference was statistically insignificant $(2.6 \pm 0.6$ vs $2.9 \pm 0.3, p=0.11)$. (B) The extent of the hemorrhagic lesions was broader in the treatment group, but the difference was not statistically significant $(45.0 \pm 45.5$ vs $43.0 \pm 33.8, \mathrm{p}=0.88)$. 
A

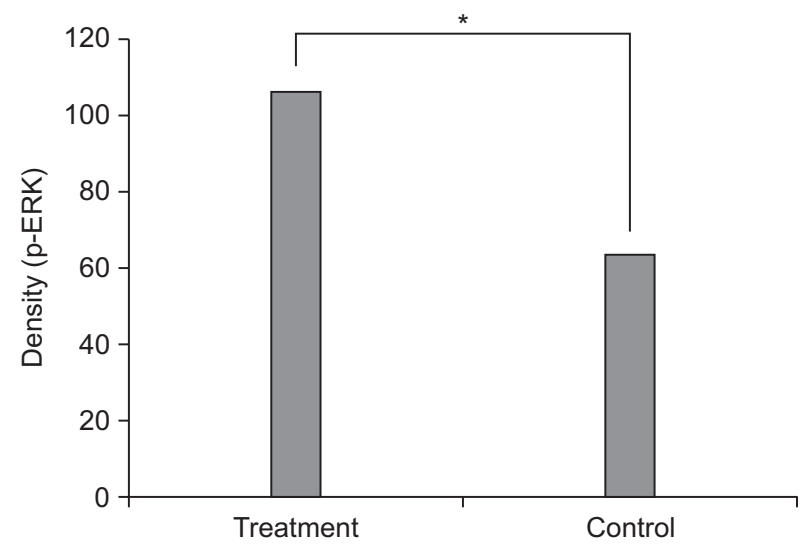

B

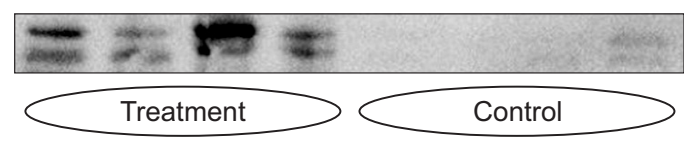

Fig. 4. (A) The densitometric assessment showed that pretreatment with DA-6034 before indomethacin increased extracellular signalregulated kinase (ERK) expression $(107.4 \pm 40.1$ vs $64.2 \pm 20.4, \mathrm{p}<0.05)$. (B) The expression of the ERK pathway was more prominent in the treatment group than in the control group. ${ }^{*} \mathrm{p}<0.05$.

$(16,204.2 \pm 13,832.1$ vs 30,403.7 $\pm 24,012.6, p<0.05)$ (Fig. 3).

\section{Effect of DA-6034 on ERK phosphorylation}

The densitometric assessment showed that pretreatment with DA-6034 before indomethacin increased the ERK expression $(107.4 \pm 40.1$ vs $64.2 \pm 20.4, p<0.05)$ (Fig. $4 A$ ). The expression of ERK pathway was more prominent in the treatment group compared to that of the control group (Fig. 4B). As such, the intestinal permeability was negatively correlated with density of ERK expression ( $r=-0.309, p=0.096$ ) (Fig. 5).

\section{DISCUSSION}

Long term use of NSAID is known to increase intestinal permeability by inhibiting oxidative phosphorylation in intestinal epithelium and by suppressing cyclooxygenase isoenzymes. ${ }^{4}$ In patients with chronic liver disease, small intestinal bacterial overgrowth is associated with the increase of intestinal permeability. In the pathogenesis of IBD, the bacterial translocation caused by the increase of intestinal permeability leads to chronic intestinal inflammation and eventually IBD. The intestinal permeability contributes to the IBS, obesity, and insulin resistance. With this clinical awareness, studies to treat those diseases by alleviating the increase in intestinal permeability are undergoing in many perspectives. Probiotics showed an improving effect on intestinal permeability by alleviating small intestinal overgrowth in patients with chronic liver disease. Nonetheless, the anti-transforming growth factor blocker and prednisolone

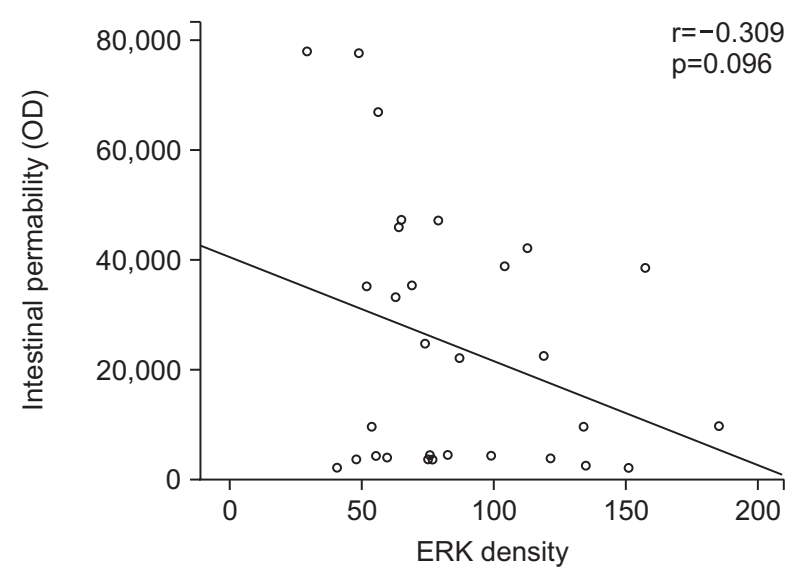

Fig. 5. Intestinal permeability was negatively correlated with the level of extracellular signal-regulated kinase (ERK) expression, suggesting that the ERK pathway is related to a protective effect on intestinal permeability $(r=-0.309, \mathrm{p}=0.096)$.

demonstrated an improving effect on intestinal permeability in IBD. ${ }^{3,16}$ Moreover, the 5-hydroxytryptamine receptor antagonist also alleviated intestinal inflammation by inhibiting inflammatory cytokine, free radical production which were effective in preventing the increase of intestinal permeability. ${ }^{17}$

The DA-6034 exhibited a gastro-protective effect in several gastric injury models, which may result from increases in defense factors, such as mucus secretion and endogenous prostaglandin E2 synthesis. It also showed anti-inflammatory effect by inhibiting $N F-\kappa B$ in colitis model, as such, it is expected to be effective in the treatment of the IBD. ${ }^{12,13}$ But DA-6034 effect on intestinal permeability remained unevaluated. This study showed the effect of DA-6034 treatment on intestinal permeability, using FITC-labeled dextran, that the intestinal permeability was lower in the treatment group compared to control group $(16,204.2 \pm 13,832.1$ vs $30,403.7 \pm 24,012.6, p<0.05)$, suggesting DA-6034 prevents the increase of intestinal permeability. In the histologic evaluation, the inflammation score in the treatment group was lower compared with that in the control group with little statistical significance $(2.61 \pm 0.60$ vs $2.88 \pm 0.33, p=0.11)$. The result did not show the significant histological protective effect of DA-6034 on intestinal damage.

Concerning the role of the ERK signaling pathway in epithelial restitution, several studies have been conducted in which activation of the ERK signaling pathway played a significant role in epithelial wound closure. ${ }^{18-20}$ We assumed ERK pathway may contribute to preventing increment of intestinal permeability. The ERK expression was compared in each group to determine whether ERK signaling pathway played a role in the underlying mechanism of DA-6034 by Western blot. The result showed that the intestinal permeability was lower in the treatment group compared to control group, ERK phosphorylation was enhanced in the treatment group, and intestinal permeability was negatively correlated with ERK phosphorylation despite of statistical 
insignificance. That implies ERK pathway takes a crucial role in preventing the increase of intestinal permeability when intestinal mucosal becomes disintegrated by indomethacin.

It is suggested that an increase in the permeability of small intestinal mucosa was an important factor in the mechanism of NSAID-induced small intestinal injury. Increased intestinal permeability leads to large molecules contained in food substances, bile acids, pancreatic juice, bacteria and other toxins within the lumen passing through the intestinal epithelial barrier, causing intestinal inflammation and injury. Therefore, the change in small intestinal permeability could directly reflect small intestinal mucosal integrity and barrier function and help to identify early damage to intestinal mucosa. ${ }^{9}$ In this study, however, improvement of intestinal permeability did not result in protection of intestinal mucosal damage. Though it has somewhat limited value for direct comparison since there were no report evaluating the effect of DA-6034 on NSAID-induced small intestinal damage with microscope, DA-6034 (30 mg/ $\mathrm{kg}$ ) is previously reported to have had protective effect on mucosal damage in dextran sulfate sodium-induced colitis model. ${ }^{13}$ Therefore, further study with modification of treatment duration, such as two or three day additional treatment after indomethacin on the effectiveness of DA-6034 in the alleviation of small intestinal mucosal damage may be necessary. In conclusion, DA-6034 shows possible role in prevention of the leaky gut syndrome in indomethacin-induced intestinal injury model by enhancing restitution of intestinal epithelial cells via ERK activation. And our results suggest DA-6034 could be a potential therapeutic drug for diseases associated with leaky gut syndrome, such as IBD, IBS, and obesity and so forth, by modifying intestinal permeability.

\section{CONFLICTS OF INTEREST}

No potential conflict of interest relevant to this article was reported.

\section{ACKNOWLEDGEMENTS}

This work was supported by a research fund from Hanyang University (HY-2011-C).

\section{REFERENCES}

1. Barbara G, Zecchi L, Barbaro R, et al. Mucosal permeability and immune activation as potential therapeutic targets of probiotics in irritable bowel syndrome. J Clin Gastroenterol 2012;46 Suppl:S52S55.

2. Gecse K, Róka R, Séra T, et al. Leaky gut in patients with diarrheapredominant irritable bowel syndrome and inactive ulcerative colitis. Digestion 2012;85:40-46.

3. Suenaert P, Bulteel V, Lemmens L, et al. Anti-tumor necrosis fac- tor treatment restores the gut barrier in Crohn's disease. Am J Gastroenterol 2002;97:2000-2004.

4. Thiéfin G, Beaugerie L. Toxic effects of nonsteroidal antiinflammatory drugs on the small bowel, colon, and rectum. Joint Bone Spine 2005;72:286-294.

5. Sandek A, Rauchhaus M, Anker SD, von Haehling S. The emerging role of the gut in chronic heart failure. Curr Opin Clin Nutr Metab Care 2008;11:632-639.

6. Ilan Y. Leaky gut and the liver: a role for bacterial translocation in nonalcoholic steatohepatitis. World J Gastroenterol 2012;18:2609 2618.

7. Boroni Moreira AP, Fiche Salles Teixeira T, do C Gouveia Peluzio M, de Cássia Gonçalves Alfenas R. Gut microbiota and the development of obesity. Nutr Hosp 2012;27:1408-1414.

8. Caricilli AM, Saad MJ. The role of gut microbiota on insulin resistance. Nutrients 2013;5:829-851.

9. Diao L, Mei Q, Xu JM, et al. Rebamipide suppresses diclofenacinduced intestinal permeability via mitochondrial protection in mice. World J Gastroenterol 2012;18:1059-1066.

10. Pozzoli C, Menozzi A, Grandi D, et al. Protective effects of proton pump inhibitors against indomethacin-induced lesions in the rat small intestine. Naunyn Schmiedebergs Arch Pharmacol 2007;374:283-291.

11. Ukena SN, Singh A, Dringenberg U, et al. Probiotic Escherichia coli Nissle 1917 inhibits leaky gut by enhancing mucosal integrity. PLoS One 2007;2:e1308.

12. Kim JW, Kim BG, Lee KL, et al. The effects of DA-6034 on NFkappaB activity induced by lipopolysaccharide or tumor necrosis factor alpha in a human colonic epithelial cell line. Hepatogastroenterology 2008;55:2059-2064.

13. Nam SY, Kim JS, Kim JM, et al. DA-6034, a derivative of flavonoid, prevents and ameliorates dextran sulfate sodium-induced colitis and inhibits colon carcinogenesis. Exp Biol Med (Maywood) 2008;233:180-191.

14. Garg P, Rojas M, Ravi A, et al. Selective ablation of matrix metalloproteinase-2 exacerbates experimental colitis: contrasting role of gelatinases in the pathogenesis of colitis. $\mathrm{J}$ Immunol 2006;177:4103-4112.

15. Chiu CJ, McArdle AH, Brown R, Scott HJ, Gurd FN. Intestinal mucosal lesion in low-flow states: I. a morphological, hemodynamic, and metabolic reappraisal. Arch Surg 1970;101:478-483.

16. Miki K, Moore DJ, Butler RN, Southcott E, Couper RT, Davidson GP. The sugar permeability test reflects disease activity in children and adolescents with inflammatory bowel disease. J Pediatr 1998;133:750-754.

17. Kato S, Matsuda N, Matsumoto K, et al. Dual role of serotonin in the pathogenesis of indomethacin-induced small intestinal ulceration: pro-ulcerogenic action via 5-HT3 receptors and anti-ulcerogenic action via 5-HT4 receptors. Pharmacol Res 2012;66:226234.

18. Engelman JA. Targeting PI3K signalling in cancer: opportunities, challenges and limitations. Nat Rev Cancer 2009;9:550-562. 
19. Suzuki H, Masaoka T, Minegishi Y, Motosugi Y, Miura S, Ishii H. Lansoprazole promotes gastric mucosal cell proliferation and migration by activating p44/p42 mitogen-activated protein kinase.
Wound Repair Regen 2004;12:93-99.

20. Lee SH. Intestinal permeability regulation by tight junction: implication on inflammatory bowel diseases. Intest Res 2015;13:11-18. 